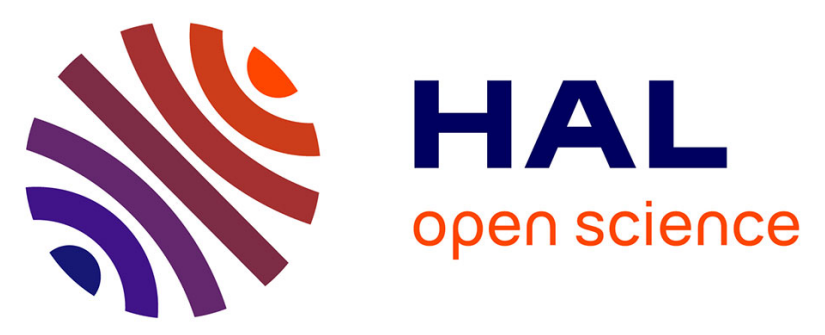

\title{
Influence of doping level and surface states in tunneling spectroscopy of an In0.53Ga0.47As quantum well grown on p-type doped $\operatorname{InP}(001)$
}

\author{
Nathali Alexandra Franchina Vergel, A. Tadjine, V. Notot, M. Mohr, A. \\ Kouassi N'guissan, Christophe Coinon, Maxime Berthe, Louis Biadala, K. \\ Sossoe, M. Dzagli, et al.
}

\section{To cite this version:}

Nathali Alexandra Franchina Vergel, A. Tadjine, V. Notot, M. Mohr, A. Kouassi N'guissan, et al.. Influence of doping level and surface states in tunneling spectroscopy of an In0.53Ga0.47As quantum well grown on p-type doped InP(001). Physical Review Materials, 2019, 3 (9), 10.1103/PhysRevMaterials.3.094604 . hal-02309916

\section{HAL Id: hal-02309916 https://hal.science/hal-02309916}

Submitted on 20 Nov 2020

HAL is a multi-disciplinary open access archive for the deposit and dissemination of scientific research documents, whether they are published or not. The documents may come from teaching and research institutions in France or abroad, or from public or private research centers.
L'archive ouverte pluridisciplinaire HAL, est destinée au dépôt et à la diffusion de documents scientifiques de niveau recherche, publiés ou non, émanant des établissements d'enseignement et de recherche français ou étrangers, des laboratoires publics ou privés. 


\title{
Influence of doping level and surface states in tunneling spectroscopy of an $\operatorname{In}_{0.53} \mathbf{G a}_{0.47} \mathrm{As}$ quantum well grown on $p$-type doped InP(001)
}

\author{
N. A. Franchina Vergel, ${ }^{1}$ A. Tadjine,${ }^{1}$ V. Notot, ${ }^{1}$ M. Mohr,${ }^{2}$ A. Kouassi N'Guissan, ${ }^{3}$ C. Coinon, ${ }^{1}$ M. Berthe, ${ }^{1}$ L. Biadala,${ }^{1}$ \\ K. K. Sossoe, ${ }^{3}$ M. M. Dzagli, ${ }^{3}$ J.-C. Girard, ${ }^{4}$ G. Rodary, ${ }^{4}$ L. Desplanque, ${ }^{1}$ R. Berndt, ${ }^{2}$ D. Stiévenard, ${ }^{1}$ \\ X. Wallart, ${ }^{1}$ C. Delerue, ${ }^{1}$ and B. Grandidier $\oplus^{1, *}$ \\ ${ }^{1}$ Université Lille, CNRS, Centrale Lille, ISEN, Université Valenciennes, UMR 8520 - IEMN, F-59000 Lille, France \\ ${ }^{2}$ Institut für Experimentelle und Angewandte Physik, Christian-Albrechts-Universität zu Kiel, D-24098 Kiel, Germany \\ ${ }^{3}$ Laboratoire de Physique des Matériaux et Composants à Semi-Conducteurs (LPMCS), Département de Physique, FDS, \\ Université de Lomé, 01 BP 1515 Lomé 1, Togo \\ ${ }^{4}$ Centre de Nanosciences et de Nanotechnologies (C2N), UMR 9001 CNRS, University Paris Sud, University Paris-Saclay, \\ 10 bd Thomas Gobert, 91120 Palaiseau, France
}

(Received 10 May 2019; revised manuscript received 25 August 2019; published 20 September 2019)

\begin{abstract}
A steplike density of states is the hallmark of a two-dimensional electron gas. Here, scanning tunneling spectroscopy is used to investigate this property in the differential conductance of an $\operatorname{In}_{0.53} \mathrm{Ga}_{0.47} \mathrm{As}$ quantum well grown on $p$-type doped $\operatorname{InP}(001)$. We show that the appearance of a steplike function in the conduction band strongly depends on both the hole concentration in the quantum well and the current injected into the quantized states. Based on four-point probe transport measurements and tunneling-induced light-emission spectroscopy, we discuss the physical mechanisms at the origin of the current and unveil the significant contribution of midgap surface states in the relaxation of the tunneling electrons. These surface states, via pinning of the Fermi level, also affect the potential landscape across the quantum well, as demonstrated by tight-binding calculations of the quantum well electronic structure.
\end{abstract}

DOI: 10.1103/PhysRevMaterials.3.094604

\section{INTRODUCTION}

Scanning tunneling spectroscopy (STS) can provide a direct measurement of the electronic local density of states (LDOS) in quantum semiconductor structures [1-3]. In particular, when the electrons are confined in a quantum well, the density of states exhibits a sequence of steps that was directly observed in the conductance spectra of cleaved III-V semiconductor quantum wells, each step corresponding to an electron subband in the conduction band [4-6]. Crucial to the successful observation of a two-dimensional electron gas is the absence of surfaces states in the energy range corresponding to the electron subbands, a phenomenon which occurs when III-V zinc-blende semiconductors are cleaved to expose a (110) surface. This natural passivation repels the surface states far away from the band edges, allowing a direct tunneling into bulk states such as those corresponding to the low-lying states of the electron gas.

Even in the absence of surface states, the observation of the two-dimensional density of states (2D-DOS) may not be straightforward. First, the probability amplitude distribution varies from one subband to the other one. If the maximum of the distribution of a subband is too deep below the passivated surface, the contribution of this subband to the tunneling current becomes negligible due to the exponential decay of the current with the distance between the scanning tunneling microscopy (STM) tip and the surface of the sample [7].

*Corresponding author: bruno.grandidier@isen.iemn.univ-lille1.fr
Fluctuations in the distribution of subsurface dopants or point defects also affect the surface potential and may prevent the observation of the 2D-DOS. Next, the electric field induced by the STM tip can penetrate into the semiconductor and locally modify the band. Depending on the tip work function and shape, this effect may lead to the formation of a tip-induced quantum dot with additional peaks superimposed to the $2 \mathrm{D}$ DOS, which differs from the expected steplike function [8]. Finally, the observation of a 2D-DOS not only depends on the injection of the current into the related states of the electron gas but also on how the electrons escape. Confining the electrons in a quantum well implies the existence of potential barriers. When the transmission probability through the potential barriers is high enough, a 2D-DOS is probed [9]. But when the transfer rate of the electron out of the quantum wells is similar to or smaller than the tunneling rate from the tip, the characteristic steplike LDOS may be lost due to charging.

In this work, we study the LDOS of an InGaAs quantum well grown on top of a $p$-type InP (001) substrate with STM. The $\operatorname{In}_{0.53} \mathrm{Ga}_{0.47}$ As ternary compound semiconductor lattice matched to InP is widely used as a channel for high-frequency III-V metal oxide semiconductor field effect transistors (MOSFETs) due to its high electron mobility [10]. Moreover, it is a type-I heterostructure, where both the electrons and the holes are confined in the quantum well, producing light emission in the wavelength range from 1.3 to $1.6 \mu \mathrm{m}$. As these wavelengths are close to the minimum attenuation and dispersion in optical fibers, it is also a key material for fiber-optic communication systems [11,12]. We 


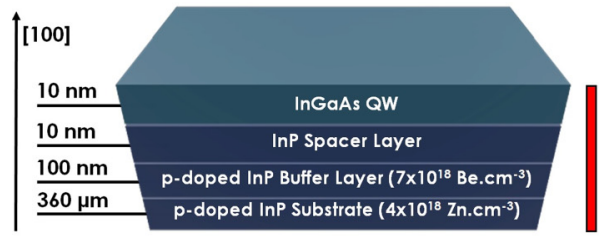

doped

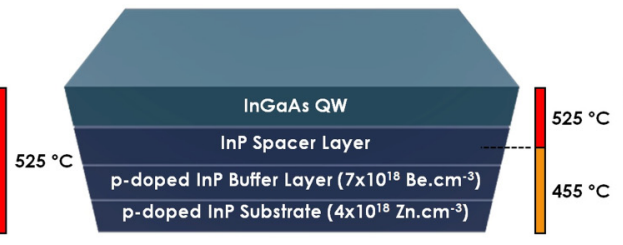

semidoped

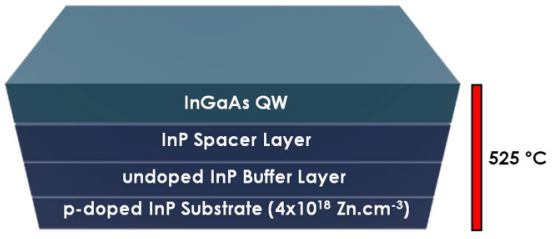

undoped

FIG. 1. Layer structure of the $\mathrm{In}_{0.53} \mathrm{Ga}_{0.47}$ As quantum wells grown on $\mathrm{InP}(001)$ by molecular beam epitaxy. The doping of the InP buffer layer and the occurrence of an interruption step during the growth of the heterostructure to change the sample temperature allows growth of quantum wells with different hole doping levels. Depending on the degree of Be diffusion during the growth, the samples are labeled doped, semidoped, and undoped.

show that scanning tunneling spectroscopy performed on the InGaAs (001) surface can reveal the 2D-DOS of the quantum well. However, the spectral shape is found to strongly depend on the doping level and the tunneling current. At high doping level and low tunneling setpoint currents, three steps in the DOS are observed in the conduction band of the quantum well, consistent with tight-binding calculations, in which the potential confinement is modified from a square to a triangular well due to a pinning of the Fermi level by midgap surface states. For decreasing doping levels, the observation of the steplike function becomes erratic and even disappears. At the lowest doping level, a new peak occurs close to the Fermi level. Based on STM-induced luminescence experiments and four-point probe STM transport measurements, we are able to rationalize this behavior and explain the charge-transport mechanisms that account for the presence of a 2D-DOS in the tunneling spectra. These experiments highlight the importance of surface states in the relaxation of tunneling electrons.

\section{EXPERIMENTAL AND THEORETICAL DETAILS}

$\mathrm{In}_{0.53} \mathrm{Ga}_{0.47} \mathrm{As} / \mathrm{InP}$ quantum well (QW) heterostructures were grown by molecular beam epitaxy on a $\mathrm{Zn} p$-type doped InP (001) substrate with a concentration of $4 \times$ $10^{18} \mathrm{~cm}^{-3}$. The samples (Fig. 1) consist of a top 10-nmthick $\operatorname{In}_{0.53} \mathrm{Ga}_{0.47}$ As quantum well, a 10 -nm-thick InP spacer layer, and a 100-nm-thick undoped/ $p$-doped InP buffer layer. Three samples with different Be dopant concentrations were prepared. For the sample denoted "doped," the whole structure was grown at $525^{\circ} \mathrm{C}$. This high temperature favors the diffusion of beryllium in the quantum well [13]. In order to reduce the amount of dopants in the quantum well, a second structure, the "semidoped" structure, was prepared at a lower temperature of $455^{\circ} \mathrm{C}$ up to the half part of the InP spacer layer. The growth was then interrupted and the temperature was raised to $525{ }^{\circ} \mathrm{C}$ before growing the $\mathrm{In}_{0.53} \mathrm{Ga}_{0.47} \mathrm{As} / \mathrm{InP}$ heterointerface. As to the third sample, labeled the "undoped" sample, the growth temperature was set at $525{ }^{\circ} \mathrm{C}$ and no dopant was intentionally incorporated during the growth. At the end of the growth, a 10-nm-thick amorphous As layer was deposited on top of the samples to protect their surface from air exposure. In order to measure the dopant concentrations in these samples with secondary ion mass spectrometry (SIMS), similar samples were grown. But instead of capping the heterostructure with a thin As layer, a 10-nm-thick undoped InP layer was grown on top of the $\operatorname{In}_{0.53} \mathrm{Ga}_{0.47}$ As quantum well in order to minimize SIMS artefacts during the initial stage of the sputtering process [14].

As the temperature used to desorb the As capping layer of an InGaAs (001) surface is known to affect the surface reconstruction [15], arsenic decapping tests were directly done after the deposition of the protective layer and monitored using reflection high-energy electron diffraction (RHEED). In this way, we were able to follow the temperature dependence of the surface reconstruction. At $340{ }^{\circ} \mathrm{C}$, the surface exhibited a $(2 \times 4)$ As reconstruction. This As-rich surface reconstruction survived up to $445^{\circ} \mathrm{C}$ before being replaced by the group-III rich $(4 \times 2)$ reconstruction. In order to limit the diffusion of impurities, the As protective layer was decapped at a low temperature of $350{ }^{\circ} \mathrm{C}$ for $2 \mathrm{~h}$, thus yielding a $(2 \times 4)$ As reconstruction. Any modification of the surface properties due to this annealing step was further analyzed with ultraviolet photoelectron spectroscopy (UPS). This study was performed with a discharge lamp operating at $21.2 \mathrm{eV}$ (He-I) in the same ultrahigh vacuum (UHV) system as the growth chamber. As shown in Fig. 2, both UPS spectra measured on the semidoped sample prior to the As capping or after the sublimation of the As capping layer exhibit a Fermi-level pinning $0.50 \pm 0.05 \mathrm{eV}$ above the top of the valence band

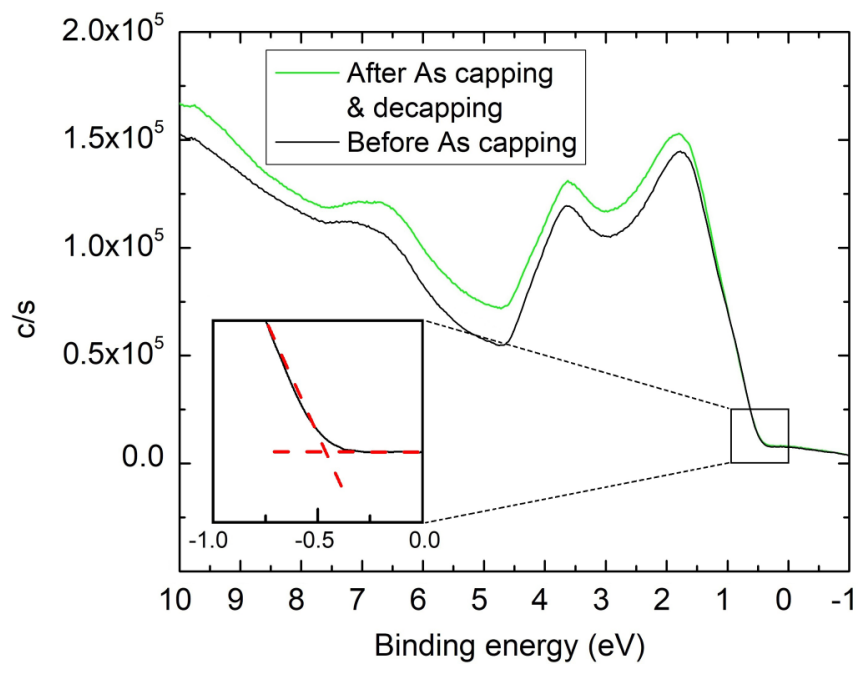

FIG. 2. UPS spectra acquired before the As capping (black line) and after the As decapping (green line). Inset: High magnification of the valence band edge. The top of the valence band is given by the intersection of the dashed straight lines. 
edge. A stable position of the Fermi level is consistent with the preservation of the $(2 \times 4)$ As reconstruction. It also indicates that the dopant concentration in the quantum well is certainly not affected by the annealing, since a significant diffusion of $p$-type dopants close to the surface is known to shift the Fermi level towards the valence band edge, despite a high concentration of surface states in the bulk band gap [16].

STM experiments were performed in four different UHV systems. Low-temperature (LT) STM measurements were done at 77 or $5 \mathrm{~K}$ with an Omicron LT-STM and an Unisoku USM 1300 LT-STM. In these systems, the differential conductance was acquired with a lock-in amplifier using a bias modulation of $10 \mathrm{mV} \mathrm{rms}$ at a frequency of $490 \mathrm{~Hz}$. The apparent band gap was measured by plotting the differential conductance in a $\log$ scale to precisely determine the noise level. Light-emission spectroscopy with STM (STM-LE) was done at $5 \mathrm{~K}$ combining a home-built LT-STM with a system of lenses mounted inside the vacuum chamber [17]. The collected tip-sample junction emission was then guided via an optical fiber to a spectrometer (Horiba iHR 320) and a liquid-nitrogen-cooled CCD detector (Horiba Symphony II CCD). A four-probe STM (Nanoprobe, Omicron) that was operated at room temperature with a control system able to run four STM tips independently was used to measure the DC conductivity of the InGaAs QW. For these measurements, the tips were positioned in a linear configuration, either with equidistant tip spacing or with a linear nonequidistant probe arrangement, in order to minimize the number of tips to be repositioned at the surface of the $\operatorname{In}_{0.53} \mathrm{Ga}_{0.47}$ As quantum well [18]. Prior to all the characterizations performed with the different STM setups, the As capping layer was sublimated in a connected preparation chamber using the temperature and time given above.

The calculations of the electronic structure of the $\mathrm{In}_{0.53} \mathrm{Ga}_{0.47} \mathrm{As} / \mathrm{InP}$ heterostructure were performed using a tight-binding approach. The $\mathrm{In}_{0.53} \mathrm{Ga}_{0.47}$ As alloy was considered as a virtual crystal in which a virtual atom (Va) replaces each In or Ga atom. Each atom in the heterostructure (Va, As, In, P) was described by 20 atomic orbitals, $s p^{3} d^{5} s^{*}$ for each spin orientation. We used the tight-binding parameters of Ref. [19] which give an energy gap of $816 \mathrm{meV}$ for the $\mathrm{In}_{0.53} \mathrm{Ga}_{0.47}$ As bulk, consistent with Ref. [20]. Based on the literature [21], we assumed a conduction-band offset between $\mathrm{In}_{0.53} \mathrm{Ga}_{0.47} \mathrm{As}$ and $\mathrm{InP}$ of $204 \mathrm{meV}$ and an infinite potential barrier at the vacuum $/ \mathrm{In}_{0.53} \mathrm{Ga}_{0.47} \mathrm{As}$ interface. Free surfaces were saturated by pseudohydrogen atoms described by a single $s$ orbital.

\section{RESULTS AND DISCUSSION}

\section{A. Spectral shape of the $I(V)$ characteristics}

Figure 3(a) shows a typical STM topograph of the $\mathrm{In}_{0.53} \mathrm{Ga}_{0.47}$ As (001) surface. The large-scale image reveals jagged terraces with an average width of $40 \mathrm{~nm}$. The measured step height is around $2.9 \AA$, corresponding to an atomic bilayer thickness. At higher resolution [Fig. 3(b)], rows of bright protrusions are clearly resolved. As depicted in the structural model of Fig 3(c), the largest protrusions correspond to two adjacent As dimers. The trenches between the rows are caused
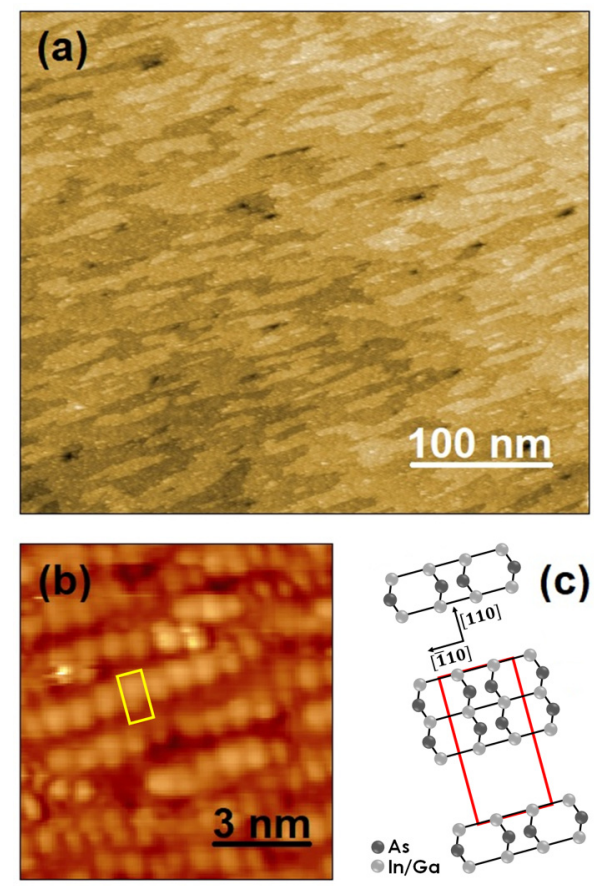

FIG. 3. (a) Large-scale STM image of the (001) surface of the $\mathrm{In}_{0.53} \mathrm{Ga}_{0.47} \mathrm{As} / \mathrm{InP}$ heterostructure. (b) High-resolution STM image. Feedback parameters: $V_{s}=+1.8 \mathrm{~V}$ and $I_{t}=10 \mathrm{pA}, T=77 \mathrm{~K}$. (c) Structural model of the $\operatorname{In}_{0.53} \mathrm{Ga}_{0.47} \mathrm{As}(001)-(2 \times 4)$ surface reconstruction with the rectangular unit cell.

by missing dimers. The zigzag structure indicates that the smallest protrusions are single As dimers, located either on the left or the right of a row. This reconstruction is typical of the As-rich $(2 \times 4)$ structure observed for the $\operatorname{In}_{0.53} \mathrm{Ga}_{0.47} \mathrm{As}$ (001) surface [15].

Tunneling spectroscopy was first performed on the doped sample [Fig. 4(a)]. In the spectra, the valence band states are probed at negative bias. The Fermi-level $E_{F}$ is determined at $0 \mathrm{~V}$, and based on the onset of the valence band (labeled $E_{V}$ ), it is found to be positioned $0.17 \pm 0.03 \mathrm{eV}$ above the top of the valence band. A sequence of three steps is visible at positive voltages. Based on the position of the first step edge and the onset of the valence band, we measure an apparent band gap of $0.90 \pm 0.03 \mathrm{eV}$, which is consistent with the energy of the photoluminescence peak obtained for a 10-nm-thick $\mathrm{In}_{0.53} \mathrm{Ga}_{0.47} \mathrm{As} \mathrm{QW}$ inserted between two InP potential barriers [22,23]. In a previous report on tunneling experiments [9], the three steps were attributed to the electron subbands of the QW. Although the thickness of the $\mathrm{In}_{0.53} \mathrm{Ga}_{0.47} \mathrm{As} \mathrm{QW}$ grown on InP (111)A was identical, the potential barrier consisted of an $\operatorname{In}_{0.58} \mathrm{Al}_{0.42} \mathrm{As}$ layer, resulting in a stronger confinement in the conduction band. Moreover, the doping of the $\mathrm{InP}$ buffer was $n$ type instead of $p$ type in the present work. Therefore, to understand the origin of the three steps, we assessed the confinement potential profile in the heterostructure that is likely to change with the type of doping. For that purpose, we estimated the band bending at the surface of the quantum well, taking into account the proximity of the STM tip. To simplify the problem, we considered a bulk $\operatorname{In}_{0.53} \mathrm{Ga}_{0.47} \mathrm{As}$ sample and solved the Poisson equation, taking into account the 

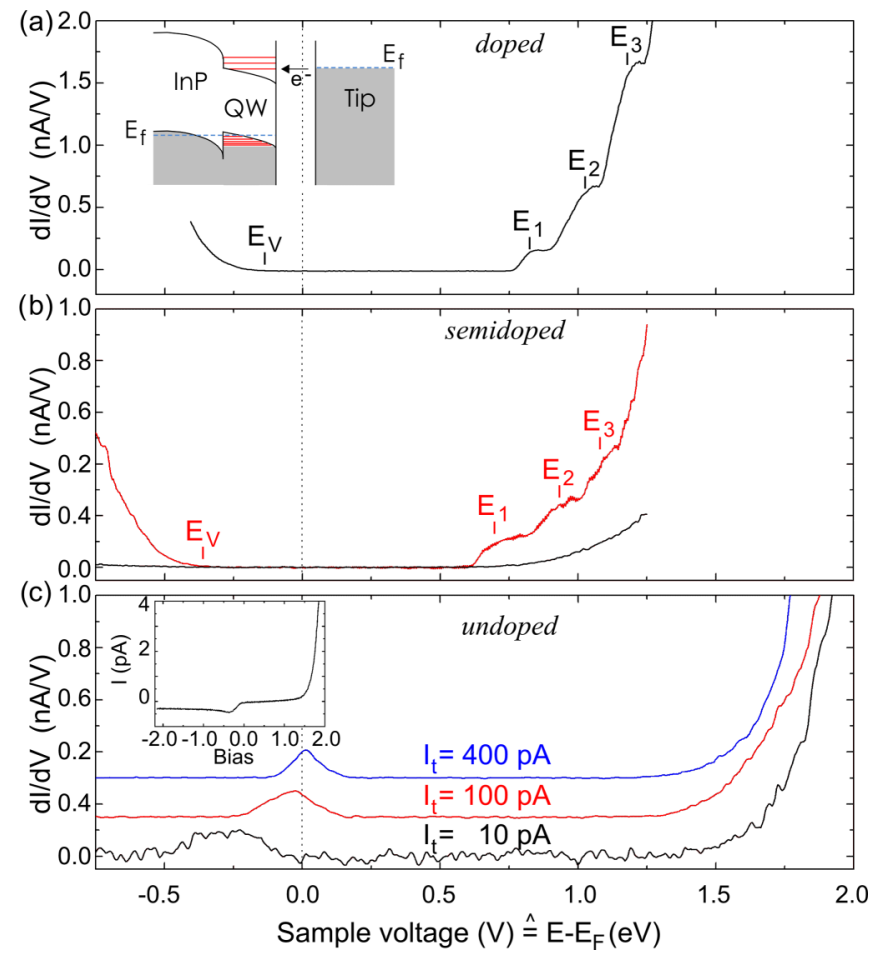

FIG. 4. $d I / d V$ spectra acquired on the (a) doped, (b) semidoped, and (c) undoped $\mathrm{In}_{0.53} \mathrm{Ga}_{0.47} \mathrm{As} / \mathrm{InP}$ heterostructures measured at $77 \mathrm{~K}$. For the semidoped sample, two different types of spectra were measured as a function of the area probed by the STM tip. For the undoped sample, the same area was probed with different setpoint currents indicated in the graph. The three electron subbands and the top of the valence band are labeled $E_{1}, E_{2}$ and $E_{3}$ and $E_{V}$ in (a) and (b). Insets: (a) Band diagram of the tunneling junction with the first electron subband aligned with the tip Fermi level $E_{f}$. (c) $I(V)$ characteristic measured at a setpoint current of 10 pA. Feedback parameters: (a) $V_{s}=+1.30 \mathrm{~V} / I_{\text {setpoint }}=400 \mathrm{pA}$, (b) $V_{s}=+1.25 \mathrm{~V} / I_{\text {setpoint }}=400 \mathrm{pA}$, and (c) $V_{s}=+2.0 \mathrm{~V}$.

boundary condition at the vacuum/semiconductor interface [24]. We assumed a Gaussian band of surface states centered $0.4 \mathrm{eV}$ above the top of the valence band with a density of $3 \times 10^{12} \mathrm{~cm}^{-2}$ that was found for a $p$-type GaAs (001) surface having the same reconstruction [16]. This density pins the Fermi level close to midgap for small doping levels, consistent with the UPS measurements of the semidoped sample. It is, however, insufficient to keep the Fermi-level midgap at higher doping levels, so that it complies with a shift of the Fermi level closer to the valence band, as the doping level in the quantum well increases. As seen in the inset of Fig. 5, taking a $p$-type doping concentration of $1 \times 10^{18} \mathrm{~cm}^{-3}$ results in a downward band bending extending over $10 \mathrm{~nm}$ in the semiconductor material, even at a positive bias of $0.8 \mathrm{~V}$. This bias corresponds to the alignment of the tip Fermi level with the first electron subband of the quantum well. A similar band profile is also found at a bias of $+1.0 \mathrm{~V}$, although the band bending is reduced by $50 \mathrm{meV}$. These results indicate that the potential distribution in the well can be considered as triangular over the whole range of positive bias used to probe the LDOS of the QW.

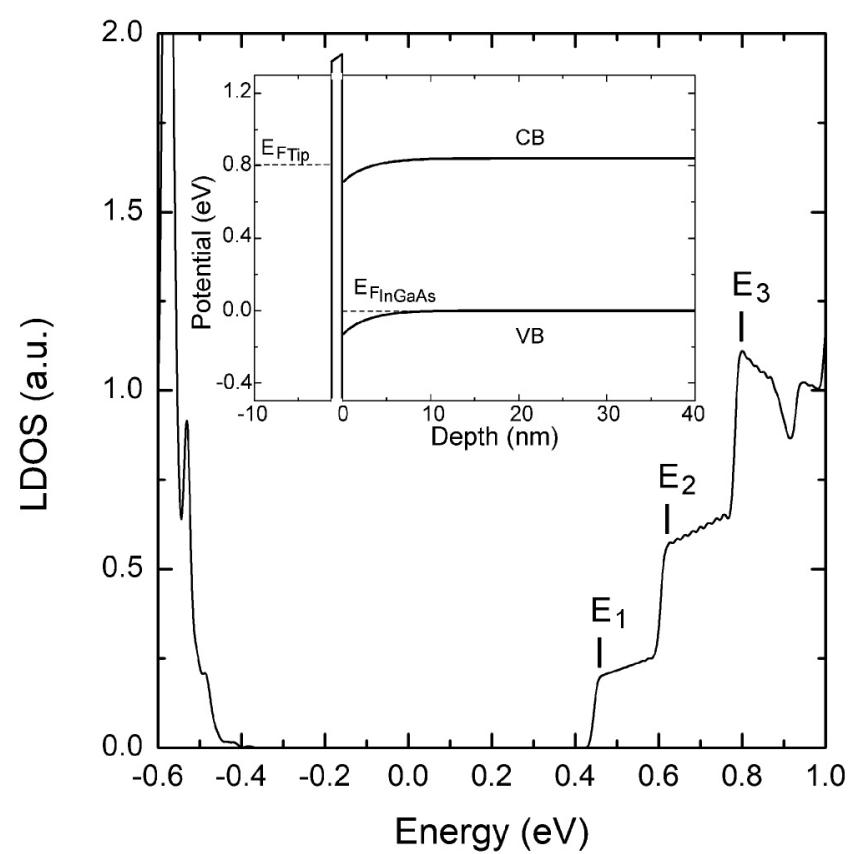

FIG. 5. Theoretical LDOS obtained from tight-binding calculations. The three electron subbands are labeled $E_{1}, E_{2}$, and $E_{3}$. Inset: Band diagram of the metal-vacuum- $\operatorname{In}_{0.53} \mathrm{Ga}_{0.47} \mathrm{As}$ at $77 \mathrm{~K}$ for a sample bias of $0.8 \mathrm{~V}$. The tip-induced band bending was calculated with the SEMITIP code [24], assuming a doping level of $1 \times 10^{18} \mathrm{~cm}^{-3}$, a tip radius of $5 \mathrm{~nm}$, a tip-sample distance of $5 \AA$, and a band of surface states positioned at an energy of $0.4 \mathrm{eV}$ above the top of the valence band with a density of $3 \times 10^{12} \mathrm{~cm}^{-2}$. The conduction and valence bands are labeled $\mathrm{CB}$ and $\mathrm{VB}$, respectively. The dashed lines indicate the positions of the Fermi level in the tip (left) and in $\mathrm{In}_{0.53} \mathrm{Ga}_{0.47} \mathrm{As}$ (right).

Therefore, the LDOS calculated within the tight-binding approximation was not only obtained for a flat potential distribution across the heterostructure but also for a triangular well. This potential shape results from the pinning of the Fermi level by the surface states existing at the vacuum $/ \mathrm{In}_{0.53} \mathrm{Ga}_{0.47} \mathrm{As}$ interface. While a flat potential distribution leads to two quantized states in the conduction band, the calculations for the triangular well reveals three steps at positive energies (Fig. 5), consistent with the STS measurements of the doped sample. We note that small oscillations in the LDOS are induced by the finite $k$ sampling in the Brillouin zone. The theoretical separation between the quantized states $E_{2}-E_{1}$ of $0.16 \mathrm{eV}$ and $E_{3}-E_{2}$ of $0.18 \mathrm{eV}$ fits well with the experimental values of 0.17 and $0.19 \mathrm{eV}$, indicating that the potential in the tip/vacuum/sample junction almost fully drops in the vacuum due to a high concentration of holes in the quantum well.

In contrast to the doped sample, where the three steps are observed on the whole surface, the semidoped sample shows two types of spectra [Fig. 4(b)]. The first one is similar to the doped sample: the differential conductance yields three steps at positive bias. We note that the step width and the apparent band gap $(1.00 \pm 0.03 \mathrm{eV})$ are slightly larger. Moreover, the Fermi-level position is shifted to a higher energy at the surface of the $\mathrm{In}_{0.53} \mathrm{Ga}_{0.47} \mathrm{As}$ quantum well. It is now $0.38 \pm 0.03 \mathrm{eV}$ 

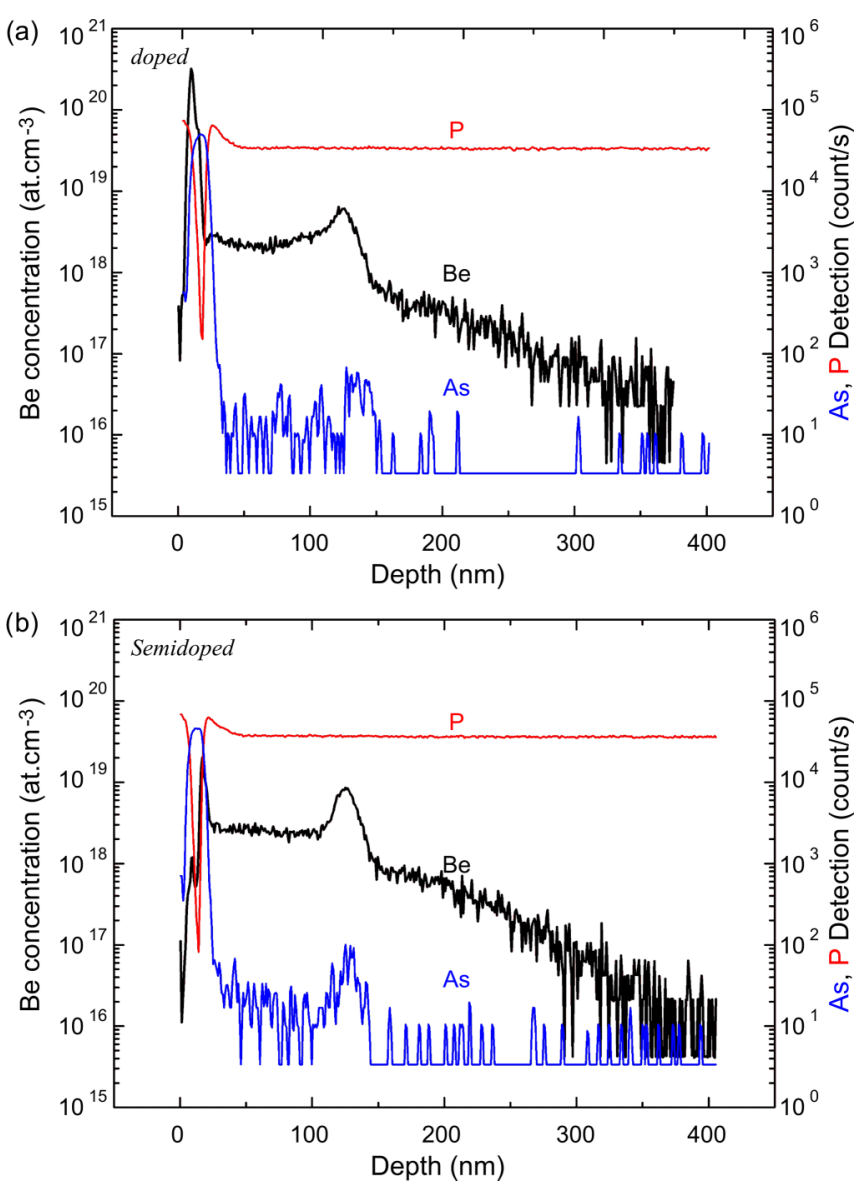

FIG. 6. Depth profiles of the Be, P, and As atomic elements in the (a) doped and (b) semidoped samples capped with a 10-nm-thick InP layer instead of the As amorphous layer.

above the onset of the valence band, closer to the energy measured with UPS in Fig. 2. As to the second type of spectra, the differential conductance does not show the steplike function expected for a quantum well at positive bias. The weaker signal measured at both positive and negative biases corresponds to an exponential increase of the current with the bias, yielding an apparent band gap of $1.20 \pm 0.03 \mathrm{eV}$.

Finally, for the undoped sample, spatially resolved tunneling spectra do not show any features in the energy range of the three steps [Fig. 4(c)]. The onset at positive bias is shifted to $1.25 \mathrm{~V}$ at a setpoint current of $400 \mathrm{pA}$. This value corresponds to the transition between the third step and the exponential increase obtained for the semidoped sample. At negative bias, no signal is measured and a peak is clearly seen in the vicinity of the Fermi level. This peak is found to shift towards the Fermi level with increasing setpoint currents and its origin will be discussed later. Based on the different spectra observed between the three samples, it is straightforward that the doping level of the structure has a direct impact on the occurrence of the 2D-DOS.

\section{B. Importance of the hole concentration in the quantum well}

Despite the similarity of the doping level incorporated during the growth of the doped and semidoped samples, the different spectra of Fig. 4 indicate a significant modification

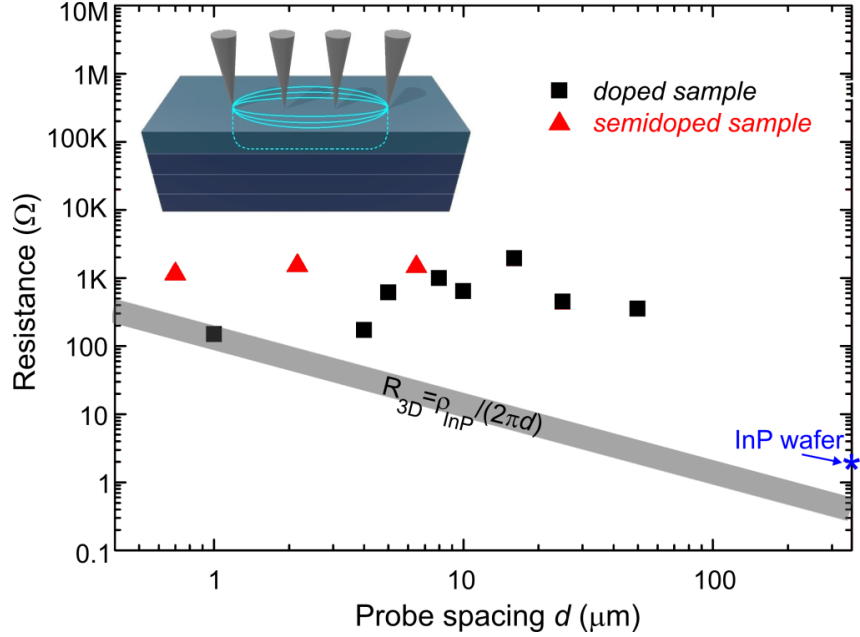

FIG. 7. Room-temperature four-point probe resistance of the $\mathrm{In}_{0.53} \mathrm{Ga}_{0.47} \mathrm{As} / \mathrm{InP}$ heterostructure for the doped and semidoped samples as a function of the probe spacing $d$. The probes are arranged in a collinear array with equidistant contact spacing, as shown by the inset. The penetration depth of the current distribution is limited to the quantum well. The light-gray band highlights the variation of the resistance for three-dimensional semi-infinite bulk InP, which is inversely proportional to the probe spacing with a bulk resistivity of $0.08 \Omega \mathrm{cm}$. The blue star shows the measured four-point probe resistance of the InP substrate only.

of the doping profile in the heterostructure. As a result, the Be concentration was analyzed by SIMS. Figure 6 reveals a strong Be dopant redistribution with respect to the intended dopant profile, since the incorporation of Be dopants during the growth occurred in the 100-nm-thick InP buffer located $30 \mathrm{~nm}$ below the surface only. In both profiles, a smaller Be concentration than $7 \times 10^{18} \mathrm{~cm}^{-3}$ was measured in this region, whereas several peaks are visible at the interfaces and surface of the sample. This redistribution agrees with previous analyses of InGaAs and InGaAs/InP heterostructures upon annealing $[13,25,26]$. It increases with higher growth temperatures and results in a much higher doping level of the QW for the doped sample in comparison with the semidoped sample, consistent with the STS findings. To ascertain the electrical activation of the Be dopants in the quantum well region, four-point probe measurements of the resistivity were performed. For the undoped sample, the absence of doping in the whole structure led to poor electrical contact between the tips and the surface, preventing the measurement of the four-probe resistance $R_{4 p}$. As to the doped and semidoped samples, we were able to measure $R_{4 p}$ as a function of the tip separation, as shown in Fig. 7. Although the data scatter more for the doped sample due to a repositioning of the tips in different areas of the sample and along different crystallographic orientations, the resistance can be considered independent of the distance between the probes [27]. This behavior is in contrast to the typical increase of the resistance for a homogeneous and isotropic thick-enough three-dimensional resistive doped $\mathrm{InP}$ semiconductor indicated by the tilted band in Fig. 7 [28]. It is the signature of a two-dimensional transport [29]. 
While the band offset is large enough to prevent the penetration of the current into the InP layers, transport involves two current paths that we consider to be spatially separated: one in the quantum well and one at its surface through the band of surface states. Thus we assume a parallel-circuit model, where the four-probe resistance $R_{4 p}$ is given by

$$
\frac{1}{R_{4 p}}=\frac{1}{R_{\mathrm{QW}}}+\frac{1}{R_{S S}},
$$

with $R_{Q W}$ the resistances of the QW and $R_{s S}$ the resistance related to the transport through the surface states. The conductivity of the quantum well varies with the hole concentration, which itself imposes the position of the Fermi level at the surface of the quantum well. Due to the pinning of the Fermilevel midgap at the surface of the semidoped sample and a hole concentration that is smaller than $1 \times 10^{19} \mathrm{~cm}^{-3}$ over a large fraction of the QW thickness, the QW of the semidoped sample is largely depleted in holes. Taking a conductance channel with a width $t$ smaller than the full width of the QW, the resistance of the $\mathrm{QW}$ is

$$
R_{\mathrm{QW}}=\frac{\ln 2}{\pi t e \mu_{p} p},
$$

where $\mu_{p}$ and $p$ are the hole mobility and the hole concentration, respectively. A hole concentration of $1 \times 10^{19} \mathrm{~cm}^{-3}$ results in a mobility of about $70 \mathrm{~cm}^{2} \mathrm{~V}^{-1} \mathrm{~s}^{-1}$ [30]. Assuming a QW width of $5 \mathrm{~nm}$ at most yields a four-probe resistance higher than $4 k \Omega$, which cannot explain the measured resistance of $1373 \Omega$. Therefore, the second channel through the band of surface states is less resistive. Neglecting the transport through the QW gives a sheet resistance $R_{\text {sheet }}=$ $\pi R_{4 p} / \ln 2$, which corresponds to a surface conductivity of $1.6 \times 10^{-4} S / \square$. This value is in the range of the typical surface conductivities measured for the $\mathrm{Ge}(001)$ and $\mathrm{Si}(001)$ surfaces consisting of rows of dimers [31] and accounts for the transport properties at the surface of the semidoped sample.

For the doped sample, the resistance $R_{4 p}$ is smaller. Due to the high doping level of the QW, as found by SIMS, the contribution of the QW cannot be neglected anymore. Also, the surface-state density should not be affected by the change of the p-type doping level [16]. Therefore, assuming a similar surface conductivity as the one found for the semidoped sample, a mean resistance $R_{4 p}$ of $661 \Omega$ yields a conductivity in the quantum well of $173 \mathrm{~S} \mathrm{~cm}^{-1}$. This high conductivity matches an average hole concentration of a few $10^{19} \mathrm{~cm}^{-3}$, for which the mobility is about $50 \mathrm{~cm}^{2} \mathrm{~V}^{-1} \mathrm{~s}^{-1}$ [30]. Such a concentration, which is smaller than the measured concentration of $\mathrm{Be}$ atoms in the $\mathrm{QW}$, suggests that the $\mathrm{Be}$ atoms that have segregated to the surface of the sample might not be electrically active.

\section{Origin of transport through the quantum states}

At this point, while a high concentration of holes in the quantum well is required to detect the 2D-DOS, its impact for the formation of a steady-state current measured through the quantum well states is not yet clear. At low temperature, a conduction-band offset around $200 \mathrm{meV}$ prevents the emission of electrons from $\mathrm{E}_{1}$ into the InP conduction band. Hence, the electrons must recombine with holes from the valence band. This effect is clearly demonstrated by the tunneling spectra of the "undoped" sample in Fig. 4(c), where the steplike function is not visible. When holes are absent, the tunneling electrons that are transferred from the tip to the first electron subband cannot recombine with holes in the quantum well, causing the charging of the quantum well. A higher voltage is thus required to transfer electrons from the tip states to the InP conduction band via the delocalized states of the quantum well. The onset of the exponential increase of the current occurs at a bias of $+1.25 \mathrm{~V}$ instead of $+0.50 \mathrm{~V}$, which corresponds to the resonance of the tip Fermi level with the bottom of the InP conduction band via the delocalized states of an uncharged quantum well.

In the doped and semidoped samples, the presence of holes in the quantum well allows a current to run through the electron subbands. With increasing setpoint currents, we expect the tip-induced band bending to be stronger, causing a local modification of the potential that is known to extend over tens of nanometers laterally [32]. This effect induces a weak confinement of $E_{1}$. Assuming a small degree of localization for $E_{1}$ allows the current injected from the STM tip via $E_{1}$ to be written as

$$
I=-e(1-f) W_{t},
$$

with $e$ the electron charge, $W_{t}$ the transmission probability per unit of time across the tunneling barrier, and $f$ the occupation probability of the quantum level. In the steady-state regime, this current is equal to the current emitted from $E_{1}$ to the valence band of the quantum well:

$$
I=-e f W_{r},
$$

where $W_{r}$ is the recombination rate of the electron on $\mathrm{E}_{1}$.

Eliminating $f$ from both equations results in the following expression for the current:

$$
I=-e \frac{1}{\left(\frac{1}{W_{t}}+\frac{1}{W_{r}}\right)} .
$$

Therefore, the current should be limited by the slowest process. As shown in Fig. 8, the differential conductance was acquired at different setpoint currents on the doped sample. The three electron subbands are visible up to $600 \mathrm{pA}$ without any energy shift in their position. Focusing on the variation of the current measured at the plateau corresponding to the ground states $\mathrm{E}_{1}$ (inset of Fig. 8), the current increases smoothly below 300 pA, more abruptly between 300 and $400 \mathrm{pA}$, and then saturates at around $10 \mathrm{pA}$, consistent with transport through a single quantum level involving two processes. As long as the probability $W_{t}$, which increases exponentially with setpoint current, is smaller than $W_{r}$, the current increases. Once it exceeds $W_{r}$, the current transferred through $\mathrm{E}_{1}$ saturates, yielding a recombination rate $W_{r}$ of one electron every $16 \mathrm{~ns}$. We note that a further increase of the setpoint current to $700 \mathrm{pA}$ causes a change of the differential conductance characteristic. At positive bias, the onset is shifted to a smaller bias, whereas at negative bias, a strong peak appears, indicating a change of the potential distribution across the heterostructure that could be caused by the proximity of the STM tip. From these observations, we infer a stronger downward band bending, which causes 


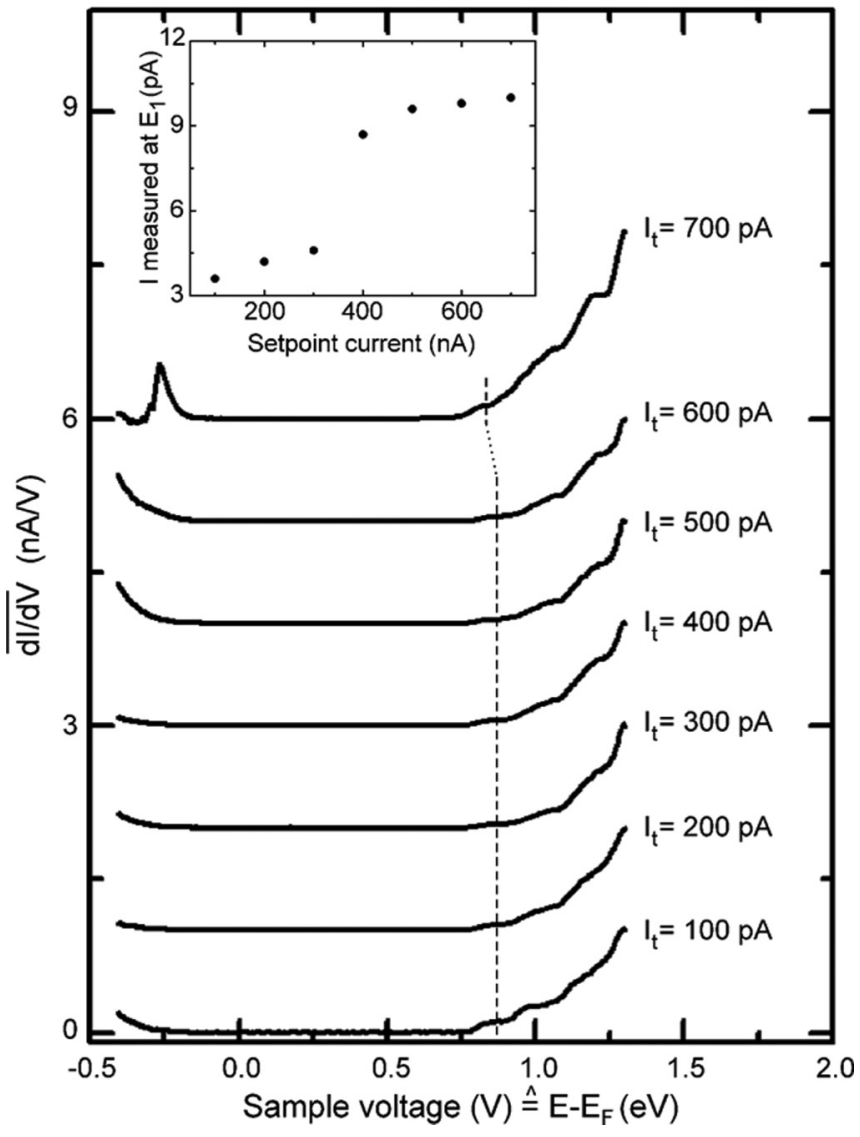

FIG. 8. Normalized $d I / d V$ spectra acquired on the doped $\mathrm{In}_{0.53} \mathrm{Ga}_{0.47} \mathrm{As} / \mathrm{InP}$ heterostructure with different setpoint currents at $77 \mathrm{~K}$. Feedback parameters: $V_{s}=+1.30 \mathrm{~V}$, the setpoint currents are indicated in the graph. Inset: Current measured as a function of the setpoint current at a bias of $+0.86 \mathrm{~V}$ between 100 and $600 \mathrm{pA}$ and $+0.83 \mathrm{~V}$ for $700 \mathrm{pA}$. These biases, highlighted by the dashed line in the main figure, correspond to the plateau of the first electron subband $\mathrm{E}_{1}$ in the $d I / d V$ characteristics.

the depletion of the wells with holes. As a result, once the tunneling electrons have left the highest occupied valence quantized states, the states become charged, which accounts for the asymmetric peak measured at negative bias.

In order to determine the nature of the recombination processes, the doped sample was analyzed with STM-LE. High currents had to be used to detect the emission of photons with energies smaller than the InP bulk band gap. The data of Fig. 9 were recorded at a sample voltage of $+3.0 \mathrm{~V}$, i.e., for electron injection, and a current of $20 \mu \mathrm{A}$. The spectrum exhibits several emission bands with photon energies smaller than the bulk band gap of InP (1.42 eV at $5 \mathrm{~K})$. The most intense band at high energy (HEB), with a maximum centered at $1.37 \mathrm{eV}$, is similar to the peak found in typical photoluminescence spectra of $p$-type InP substrates [33]. Its shape is known to change with the acceptor chemical nature, the acceptor concentration, and the excitation power $[34,35]$. The origin of the photoluminescence has been attributed to the free-to-bound exciton transition at higher energy and transitions between donor and acceptor bands at lower energy, accounting for two contributions in this band.

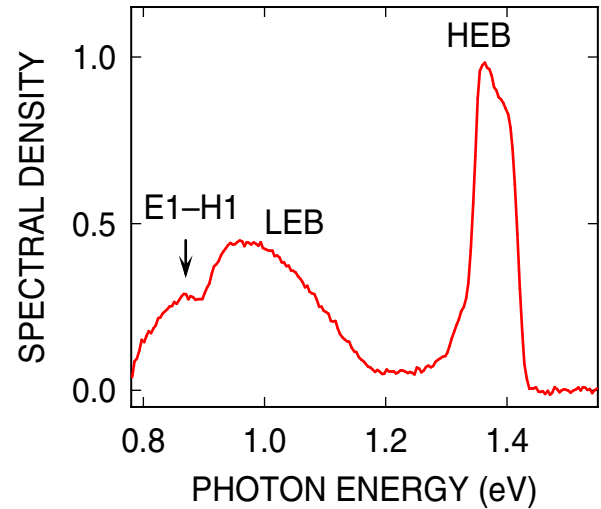

FIG. 9. Normalized tunneling-induced luminescence spectrum recorded at a sample voltage $V_{S}=+3.0 \mathrm{~V}$ and tunneling current $I=$ $20 \mu \mathrm{A}$ over an integration time of $400 \mathrm{~s}$. The spectrum is corrected for detector response. The direct transition in the $\operatorname{In}_{0.53} \mathrm{Ga}_{0.47} \mathrm{As}$ quantum well is labeled $\mathrm{E}_{1}-\mathrm{H}_{1}$, while the low and high energy bands arising from the InP layers and substrates are labeled LEB (low-energy band) and HEB (high energy band), respectively.

At lower energies, the spectrum exhibits a broad band extending from 0.9 to $1.2 \mathrm{eV}$. Comparison with photoluminescence experiments of InP substrates with a high concentration of $\mathrm{Zn}$ acceptor impurities suggests that this band is the socalled low-energy band (LEB) [36]. It was found to be stable over a wide range of excitation powers in photoluminescence [36] and has been attributed to deep levels of the $p$-type InP substrate. Conversely, the band below $0.9 \mathrm{eV}$ has never been reported from $p$-type InP. It is centered at an energy of 0.85 $\mathrm{eV}$, which matches the energy of the exciton measured in an $\mathrm{In}_{0.53} \mathrm{Ga}_{0.47} \mathrm{As} / \mathrm{InP}$ heterostructure with the same quantum well thickness [22,23]. As a result, we assign it to the radiative emission of the $\operatorname{In}_{0.53} \mathrm{Ga}_{0.47}$ As quantum well.

Comparison of the recombination rate $W_{r}$ with the exciton lifetime of similar heterostructures reveals a slightly longer lifetime with respect to a few to tens of nanoseconds range usually measured $[37,38]$. This difference could be caused by a reduced overlap between the electron and hole wave functions in the triangular well of the STM junction, with the electron localized at the surface and the hole at the $\operatorname{In}_{0.53} \mathrm{Ga}_{0.47} \mathrm{As} / \mathrm{InP}$ interface. However, the proximity of the STM tip in the light-emission experiments may modify the potential across the well. Moreover, the high current used is likely to saturate all nonradiative recombination centers at the surface, allowing a large fraction of the tunneling electrons to recombine radiatively [39]. We therefore believe that the relaxation of the tunneling electrons transferred to the conduction quantum levels also involves a nonradiative recombination process through the surface states [40,41].

While it is not clear why the surface states are not detected in the tunneling spectra measured on the doped and semidoped samples, consistent with previous studies [9,16,42], the existence of surface states clearly appears when tunneling spectroscopy is performed on the "undoped" sample. A peak is observed in the differential conductance at small negative bias in Fig. 4(c). This peak shifts towards higher energies as the tip gets closer to the surface, to become finally centered at the Fermi level. Also, the current converges towards zero 
as the sample voltage becomes more negative, as seen in the inset of Fig. 4(c). We attribute this effect to the charging of the surface states near the Fermi level. Due to the lack of enough free charge carriers in the quantum well, the electrons transferred at negative bias from the surface states to the unoccupied tip states cannot be replaced at a rate high enough, leading to positively charged surface states. Hence, the downward band bending increases, preventing the unoccupied tip states from becoming resonant with the valence band states of the quantum well for voltages higher than $-2.0 \mathrm{~V}$. In this voltage range, the number of states contributing to the current does not significantly increase, but the transmission across the tunneling barrier gets smaller as the bias decreases. This effect accounts for the $I(V)$ characteristic obtained at negative bias and demonstrates the key role of the surface states in the tunneling spectra of the "undoped" sample. We note that the significant surface conductivity of the $\operatorname{In}_{0.53} \mathrm{Ga}_{0.47} \mathrm{As}$ (001) surface could help in discharging the surface states. However, similar to the localization of the electron subbands induced by the proximity of the polarized STM tip, we expect a localization of the surface states under the STM tip that strongly decouples the states from the band of surface states.

\section{CONCLUSION}

In summary, we have investigated the density of states of an $\mathrm{In}_{0.53} \mathrm{Ga}_{0.47} \mathrm{As} \mathrm{QW}$ grown on a $p$-type doped $\mathrm{InP}$ barrier. We show that the appearance of a steplike function in the tunneling spectra, the signature of a 2D-DOS, depends on the hole concentration in the QW. As the conduction-band offset between the $\mathrm{In}_{0.53} \mathrm{Ga}_{0.47} \mathrm{As}$ quantum well and the $\mathrm{InP}$ barrier does not allow the escape of electrons from the quantized states into the InP conduction band at low temperature, the tunneling electrons recombine with the holes provided by the diffusion of Be dopant atoms during the growth. We show that two mechanisms are at work in this recombination: a radiative transition that gives rise to luminescence and a nonradiative recombination through the surface states of the quantum well. For the As-rich surface reconstruction of the $\mathrm{In}_{0.53} \mathrm{Ga}_{0.47}$ As quantum well, these surface states are located in the lowest part of the quantum well band gap, away from the conduction quantized levels, leaving the electron subbands accessible to tunneling electrons. While their presence greatly benefits the electron relaxation, they also account for the observation of three electron subbands in the conduction band of the QW. However, their density is insufficient to screen the electrical field induced by the proximity of the tip. As a result, fluctuation in the hole density at smaller Be concentrations results in stronger tip-induced band potential fluctuations at the surface of the QW, which alters the 2D-DOS and prevents the observation of the steplike function.

\section{ACKNOWLEDGMENTS}

This study was financially supported by the French National Research Agency (Dirac III-V Project No. ANR-16CE24-0007-01, Mechaspin Project No. ANR-17-CE24-002402), the EQUIPEX program Excelsior (Grant No. ANR-11EQPX-0015), and the RENATECH network.
[1] U. Banin, Y. Cao, D. Katz, and O. Millo, Nature (London) 400, 542 (1999).

[2] D. D. D. Ma, C. S. Lee, F. C. K. Au, S. Y. Tong, and S. T. Lee, Science 299, 1874 (2003).

[3] A. Urbieta, B. Grandidier, J. P. Nys, D. Deresmes, D. Stiévenard, A. Lemaître, G. Patriarche, and Y. M. Niquet, Phys. Rev. B 77, 155313 (2008).

[4] R. M. Feenstra, D. A. Collins, D. Z.-Y. Ting, M. W. Wang, and T. C. McGill, Phys. Rev. Lett. 72, 2749 (1994).

[5] K. Suzuki, K. Kanisawa, C. Janer, S. Perraud, K. Takashina, T. Fujisawa, and Y. Hirayama, Phys. Rev. Lett. 98, 136802 (2007).

[6] S. Becker, M. Liebmann, T. Mashoff, M. Pratzer, and M. Morgenstern, Phys. Rev. B 81, 155308 (2010).

[7] M. Morgenstern, A. Georgi, C. Straßer, C. R. Ast, S. Becker, and M. Liebmann, Phys. E (Amsterdam, Neth.) 44, 1795 (2012).

[8] M. Morgenstern, J. Klijn, Chr. Meyer, M. Getzlaff, R. Adelung, R. A. Römer, K. Rossnagel, L. Kipp, M. Skibowski, and R. Wiesendanger, Phys. Rev. Lett. 89, 136806 (2002).

[9] S. Perraud, K. Kanisawa, Z. Z. Wang, and T. Fujisawa, Phys. Rev. B 76, 195333 (2007).

[10] J. A. Del Alamo, Nature (London) 479, 317 (2011).

[11] G. Ribordy, N. Gisin, O. Guinnard, D. Stuck, M. Wegmuller, and H. Zbinden, J. Mod. Opt. 51, 1381 (2004).
[12] N. Namekata, S. Sasamori, and S. Inoue, Opt. Express 14, 10043 (2006).

[13] S. Koumetz, J. Marcon, K. Ketata, M. Ketata, C. DubonChevallier, P. Launay, and J. L. Benchimol, Appl. Phys. Lett. 67, 2161 (1995).

[14] P. C. Zalm, Rep. Prog. Phys. 58, 1321 (1995).

[15] J. Shen, D. L. Winn, W. Melitz, J. B. Clemens, and A. C. Kummel, ECS Trans. 16, 463 (2008).

[16] M. D. Pashley, K. W. Haberern, R. M. Feenstra, and P. D. Kirchner, Phys. Rev. B 48, 4612 (1993).

[17] G. Hoffmann, J. Kröger, and R. Berndt, Rev. Sci. Instrum. 73, 305 (2002).

[18] S. Just, M. Blab, S. Korte, V. Cherepanov, H. Soltner, and B. Voigtländer, Phys. Rev. Lett. 115, 066801 (2015).

[19] L. C. Post, T. Xu, N. A. Franchina Vergel, A. Tadjine, Y. Lambert, F. Vaurette, D. Yarekha, L. Desplanque, D. Stiévenard, X. Wallart, B. Grandidier, C. Delerue, and D. Vanmaekelbergh, Nanotechnology 30, 155301 (2019).

[20] I. Vurgaftman, J. R. Meyer, and L. R. Ram-Mohan, J. Appl. Phys. 89, 5815 (2001).

[21] P. E. Smith, S. H. Goss, M. Gao, M. K. Hudait, Y. Lin, S. A. Ringel, and L. J. Brillson, J. Vac. Sci. Technol. B 23, 1832 (2005). 
[22] K. W. Carey, R. Hull, J. E. Fouquet, F. G. Kellert, and G. R. Trott, Appl. Phys. Lett. 51, 910 (1987).

[23] E. H. Reihlen, A. Persson, T. Y. Wang, K. L. Fry, and G. B. Stringfellow, J. Appl. Phys. 66, 5554 (1989).

[24] R. M. Feenstra, J. Vac. Sci. Technol. B 21, 2080 (2003).

[25] E. G. Scott, D. Wake, G. D. T. Spiller, and G. J. Davies, J. Appl. Phys. 66, 5344 (1989).

[26] M. B. Panish, R. A. Hamm, D. Ritter, and H. S. Luftman, J. Cryst. Growth 112, 343 (1991).

[27] E. Perkins, L. Barreto, J. Wells, and P. Hofmann, Rev. Sci. Instrum. 84, 033901 (2013).

[28] I. Shiraki, F. Tanabe, R. Hobara, T. Nagao, and S. Hasegawa, Surf. Sci. 493, 633 (2001).

[29] I. Miccoli, F. Edler, H. Pfnür, and C. Tegenkamp, J. Phys.: Condens. Matter 27, 223201 (2015).

[30] S. Godey, S. Dhellemmes, A. Wilk, M. Zaknoune, and F. Mollot, J. Cryst. Growth 278, 600 (2005).

[31] S. Just, H. Soltner, S. Korte, V. Cherepanov, and B. Voigtländer, Phys. Rev. B 95, 075310 (2017).

[32] R. Dombrowski, Chr. Steinebach, Chr. Wittneven, M. Morgenstern, and R. Wiesendanger, Phys. Rev. B 59, 8043 (1999).

[33] V. Swaminathan, V. M. Donnelly, and J. Long, J. Appl. Phys. $\mathbf{5 8}, 4565$ (1985).
[34] E. W. Williams, W. Elder, M. G. Astles, M. Webb, J. B. Mullin, B. Straughan, and P. J. Tufton, J. Electrochem. Soc. 120, 1741 (1973).

[35] L. Biadala, W. Peng, Y. Lambert, J. H. Kim, D. Canneson, A. Houppe, M. Berthe, D. Troadec, D. Deresmes, G. Patriarche, T. Xu, X. Pi, X. Wallart, C. Delerue, M. Bayer, J. Xu, and B. Grandidier, ACS Nano 13, 1961 (2019).

[36] Y. Moon, S. Si, E. Yoon, and S. J. Kim, J. Appl. Phys. 83, 2261 (1998).

[37] C. H. Henry, R. A. Logan, F. R. Merritt, and C. G. Bethea, Electron. Lett. 20, 358 (1984).

[38] U. Cebulla, G. Bacher, A. Forchel, G. Mayer, and W. T. Tsang, Phys. Rev. B 39, 6257, (1989).

[39] M. Kemerink, J. W. Gerritsen, P. M. Koenraad, H. van Kempen, and J. H. Wolter, Appl. Phys. Lett. 75, 3656 (1999).

[40] R. Driad, Z. H. Lu, S. Charbonneau, W. R. McKinnon, S. Laframboise, P. J. Poole, and S. P. McAlister, Appl. Phys. Lett. 73, 665 (1998).

[41] M. J. Milla, J. M. Ulloa, and A. Guzmán, Appl. Phys. Lett. 100, 131601 (2012).

[42] S. Kasai, N. Negoro, and H. Hasegawa, Appl. Surf. Sci. 175, 255 (2001). 